\title{
Estructura factorial del Cuestionario de Apoyo Social MOS en ancianos colombianos con dolor crónico.
}

\section{Factorial structure of the MOS Social Support Survey among senior and elder Colombian population diagnosed with chronic pain.}

\author{
Marta Martín-Carbonell \\ Universidad Cooperativa de Colombia, Colombia \\ Ara Cerquera-Córdoba \\ Universidad Pontificia Bolivariana de Bucaramanga, Colombia \\ Martha Fernández-Daza \\ Juan D. Higuita \\ Gonzalo Galván Patrignani \\ Manuel Guerrero Martel \\ Universidad Cooperativa de Colombia, Colombia \\ Antonio Riquelme Marín \\ Universidad de Murcia, España
}

(Recibido: 12 de junio de 2018) (Aceptado: 26 de abril de 2019)

\begin{abstract}
Resumen
El Cuestionario de Apoyo Social MOS es uno de los instrumentos que más se utilizan en la investigación y en la práctica gerontológica, pero no hay estudios acerca de su validez para la evaluación de ancianos colombianos. El objetivo de esta investigación fue valorar si la estructura de 4 factores descrita en otras poblaciones es válida, estudiar la fiabilidad y determinar diferencias atribuibles a género y convivencia con una pareja. Se trabajó con una muestra intencional de 463 mayores, de diversas regiones de Colombia. El análisis estadístico de los ítems evidenció ausencia de normalidad y altas correlaciones inter ítems e ítemescala (superiores a .60), por lo que se seleccionó el método de Mínimos Cuadrados no Ponderados (ULS) para el análisis factorial confirmatorio, con el que se evaluaron tres modelos (de un factor, de tres y de cuatro factores correlacionados) siendo el de 4 factores el que obtuvo mejores índices (NFI=.997; AGFI=.996; $\mathrm{RMR}=.056$; NFI=.996). Los coeficientes alfa de las subescalas estaban entre .86 y .98. Se encontraron diferencias significativas en apoyo social percibido atribuibles al género y a tener pareja. Los resultados sustentan la validez del cuestionario MOS para la evaluación del apoyo social percibido por adultos mayores colombianos con dolor crónico.

Palabras claves: Apoyo social, Cuestionario de Apoyo Social MOS, Vejez, Evaluación psicológica, Propiedades psicométricas, Validez, Fiabilidad.
\end{abstract}

Correspondencia: martha.martinc@campusucc.edu.co 


\begin{abstract}
Perceived social support refers to one's confidence that social support or resources are available if needed. It is related to health and quality of life in elderly, so it is recommended to be evaluated. The Social Support Survey MOS have been used but there is no evidence about its validity in Colombian ancient people with chronic pain. The aim of this study was to assess the factorial structure and reliability for the Social Support Survey MOS among a group of elderly people with chronic muskuloesqueletal pain; furthermore, differences between gender and married were investigated. 463 old people of different regions of Colombia were assessed with MOS and with a sociodemographic interview. Cronbach ranged between .86 y .98 . Item correlations were higher than .60 . The model of 4 correlated factors showed good fit $(\mathrm{NFI}=.997, \mathrm{AGFI}=.996, \mathrm{RMR}=$ .056 y GFI $=.996$ ), as shown by similar studies in other populations. Differences in social support perceived by gender and marital status were found. The results support the validity for the MOS to evaluate social support in ancient Colombian people with chronic muskuloesqueletal pain.
\end{abstract}

Key Words: Social support, MOS Social Support Survey, Old population, Psychological evaluation, Psychometric properties, Validity, Realiability.

\title{
Introducción
}

El apoyo social percibido es uno de los factores determinantes de bienestar y calidad de vida en ancianos (Dumitrache, Rubio \& Rubio-Herrera, 2017; Martínez, Buz, Navarro \& López-Martín, 2017). Esta dimensión subjetiva del apoyo, entendida como la percepción de que se recibe y la satisfacción con el mismo, es la que, según diversos autores, tiene efectos más directos y evidentes en la salud y bienestar de los ancianos, y está asociada a mayor supervivencia (Bengston, 2018; Lyyra \& Heikkinen, 2006; Uchino, 2009).

En el caso específico de los ancianos con dolor osteomuscular crónico, la investigación del apoyo social ha sido prolífica, mostrando la importancia de tenerlo en cuenta para promover autonomía e independencia funcional (Lee, Kahana \& Kahana, 2016; Matos, Bernardes, Goubert, \& Beyers, 2017), por lo que existe consenso sobre la importancia de su evaluación para la práctica gerontológica y geriátrica (Cross-Denny \& Robinson, 2017; Riquelme-Marín, Martín-Carbonell, Ortigosa-Quiles, Meda-Lara, Pérez-Díaz \& López-Navas, 2016).

En una revisión, Terol, López, Neipp, Rodríguez, Pastor y Martín-Aragón (2004) encontraron que aunque hay numerosos instrumentos para la evaluación del apoyo social, la mayoría presenta problemas de fiabilidad y análisis psicométricos insuficientes, pocos cuestionarios han tenido una aceptación general y casi siempre son utilizados exclusivamente por quienes los diseñaron.

El cuestionario MOS de apoyo social (Medical Outcomes Study-Social Support Survey) supera las limitaciones anteriormente señaladas. Fue desarrollado por Sherbourne y Stewart en 1991 mediante un seguimiento realizado durante dos años a pacientes con patologías crónicas. Las autoras plantean que los elementos del test fueron seleccionados de forma cuidadosa, intentando que los ítems fueran cortos, fáciles de entender y restringidos a una única idea en cada caso (Sherbourne \& Stewart, 1991). Existe una versión corta que ha mostrado buenas propiedades psicométricas, pero hay consenso en que la versión de 20 ítems tiene mayor validez y fiabilidad, por lo que se recomienda (Londoño et al; 2012), ya que además es fácil de aplicar, calificar e interpretar. No sólo se usa en población adulta, pues también hay una versión para niños (Rodríguez, 2011).

Las autoras tuvieron en cuenta la naturaleza multidimensional y compleja del apoyo social, por lo que el instrumento pretende ofrecer información sobre dos importantes dimensiones del apoyo social percibido: la estructural, que indaga sobre la cantidad de proveedores de apoyo que dispone la persona (pregunta 1); y la dimensión funcional (preguntas de la 2 a la 20), que se focaliza en el grado en que las relaciones interpersonales cumplen determinadas funciones, como son proveer apoyo emocional y afectivo, proporcionar información necesaria y relevante para la persona (apoyo informacional) y prestar ayuda tangible y práctica. Teniendo en cuenta los resultados del análisis factorial, Sherbourne y Stewart decidieron unificar los ítems dirigidos a la evaluación del apoyo emocional e informativo, quedando al final 4 subescalas, para las que se pueden obtener puntuaciones independientes: apoyo emocional que incluye ítems que valoran la expresión de afecto positivo, comprensión y el estímulo a expresar los sentimientos, así como preguntas relacionadas con el apoyo informativo (la oferta de asesoramiento, información, orientación o comentarios); apoyo instrumental que valora la 
provisión de ayuda material o asistencia, interacción social positiva, que alude a la disposición de personas con las que es posible pasar ratos agradables y desarrollar actividades placenteras; y apoyo afectivo, relacionado con sentirse querido, poder recibir y brindar afecto.

El MOS ha sido validado y adaptado a múltiples países e idiomas como el chino (Yu, Lee \& Wo, 2004), portugués (Alonso, Montes, Menéndez \& Pereira, 2006), árabe (Alaloul \& Hall, 2016), francés-canadiense (Anderson, Bilodeau, Deshaies, Gilbert \& Jobin, 2005), chino mandarín (Wang, Zheng, He \& Thompson, 2013), taiwanese (Shyu, Tang, Liang \& Weng, 2006), y en población australiana (Holden, Lee, Hockey, Ware \& Dobson, 2014), entre otros.

Tiene varias versiones en castellano y es ampliamente utilizado en diversos países de Iberoamérica; como ejemplo se pueden citar estudios en España (Costa, Salamero \& Gil, 2007; Galindo, 2011; González \& Estévez, 2017); en Chile (Poblete, Glasinovic, Sapag, Barticevic, Arenas \& Padilla, 2015), Paraguay (Núñez, 2015), Puerto Rico (Rosa-Rodríguez, Negrón, Maldonado, Toledo \& Quiñones, 2015), Argentina (De la Revilla, Luna, Bailón \& Medina, 2005;), México (Martínez, Sánchez, Aguilar, Rodríguez \& Riveros, 2014; Hernández \& Ramírez, 2014), Ecuador (Muñoz, 2016; Ordoñez \& Tapia, 2016; Moreta, 2016); de parte del Uruguay se puede mencionar el trabajo de Akar (2013) y por Perú a Alva (2016) y Castillo (2016); en Bolivia se ha utilizado con adultos mayores institucionalizados (Gonzales, 2011) y en Brasil se hizo una adaptación en pacientes sobrevivientes de cáncer (Soares et al., 2012).

En Colombia ha sido utilizado por Trejos, Mosquera y Tuesca (2009) en niñez afectada con VIH/SIDA y emplearon la versión de De la Revilla et al. (2005). También ha sido empleado por Orcasita, Peralta, Valderrama y Uribe (2010) para investigar el papel del apoyo social como factor protector ante conductas de riesgo en adolescentes. Uribe, Orcasita y Aguillón (2012) también lo han utilizado en una muestra de adolescentes colombianos con conductas asociadas al bullying. Además, Orcasita, Uribe, Castellanos y Gutiérrez (2012) publicaron otro estudio en adolescentes con conductas sexuales de riesgo, así como Uribe (2016), quien ha realizado investigaciones relacionadas con la salud sexual, el apoyo social y funcionamiento familiar en universitarios.

La adaptación para adultos de Colombia la realizaron Londoño et al. (2012) con una muestra de 179 participantes de diversas ciudades de Colombia. La edad media fue de 38.4 años con una desviación típica $=15.2$, y un rango comprendido entre los 17 y los 86 años. Mediante análisis factorial confirmatorio, Londoño et al (2012) encontraron sustento para la estructura factorial de cuatro componentes que encontraron Sherbourne y Stewart (1981), así como también para una estructura factorial de tres componentes.

Otros estudios también han reportado una estructura de tres componentes para el MOS, como por ejemplo, Costa, Salamero y Gil (2007), Soares et al. (2012) en personas con cáncer; y Griep, Chor, Faerstein, Werneck, y Lopes (2005) en una muestra de más de 4000 adultos portugueses. En Perú se publicó un estudio de validación que reportó un nivel de confiabilidad alto $(\alpha=.94)$ para un modelo de tres factores (informacional, afectivo e instrumental) que explicaba el $54.24 \%$ de la varianza total (Baca, 2016). También se ha reportado mejores índices de ajuste para la estructura de 5 dimensiones que en 1991 propusieron Sherbourne y Stewart (Priede, Andreu, Martínez, Conchado, Ruiz-Torres, \& González-Blanch, 2018).

En Colombia se realizó un estudio previo de la dimensionalidad del MOS en ancianos con dolor crónico que reportó una estructura de dos factores (Palmar \& Pico, 2017) que explicaban el 70.62\% de la varianza total, pero la técnica usada fue análisis de componentes principales.

Baca (2016) plantea que las discrepancias encontradas en la estructura de las adaptaciones del MOS pueden explicarse por los métodos de análisis factorial que se hayan utilizado. Aunque en muchos estudios (fundamentalmente en Iberoamérica) para investigar la dimensionalidad del Cuestionario MOS se ha usado la técnica de Análisis de Componentes Principales (ACP) como una variante de análisis factorial, en realidad sus propósitos son diferentes. Lloret-Segura, Ferreres-Traver, Hernández-Baeza \& Tomás-Marco (2014, p. 4) plantean que utilizar el ACP como método de estimación de factores "supone ignorar el error de medida, lo que aumenta espuriamente las cargas factoriales, los porcentajes de varianza explicados por los factores, y puede producir una sobreestimación de la dimensionalidad del conjunto de items". Por esta razón, actualmente se recomienda verificar la estructura empírica apoyándose en los modelos de análisis factorial confirmatorio, pues se necesita que el test pueda medir constructos idénticos con la misma estructura a través de diferentes grupos (Herrero, 2010).

Aunque la investigación de Londoño et al (2012) utilizó el AFC, tiene como limitaciones que se realizó con una muestra relativamente pequeña proveniente de población general, a pesar de que el MOS estuvo originalmente diseñado para la evaluación personas con enfermedades crónicas específicas. Además, el método 
utilizado para realizar el AFC fue el de máxima verosimilitud, pero los autores no mencionaron análisis que corroboraran si los datos cumplían con los requerimientos de este método.

Por estas razones, parece razonable investigar si la estructura de 4 factores que sustenta la calificación del MOS es válida para la evaluación del apoyo social percibido por ancianos colombianos con dolor crónico, lo que resulta importante pues en caso contrario, el significado de las puntuaciones sería diferente y se afectaría la validez de contenido del cuestionario.

En el presente estudio también se investigó la fiabilidad y se estudiaron las diferencias en las puntuaciones de las sub-escalas atribuibles a variables sociodemográficas como género y convivencia con una pareja para obtener evidencias de validez diferencial, ya que existe abundante literatura acerca de que ambas juegan un papel importante en la manera en que las personas valoran y perciben el apoyo. Sobre este punto, pueden servir de referencia estudios que datan desde el siglo pasado, como los de Antonucci y Akiyama (1987), y Acitelli y Antonucci (1994), hasta recientes trabajos realizados en diversos contextos culturales como los de Schwartz y Litwin (2018), Tajvar, Grundy y Fletcher (2018) y Lu, Wang, Lei, Shi, Zhu y Jiang (2018).

\section{Método}

Participantes: Se trabajó con una muestra intencional, seleccionada por la facilidad para obtener la información. Se incluyeron personas con edad mayor o igual a 60 años, con capacidad y voluntad de responder los cuestionarios. Se excluyeron las personas con deterioro cognitivo o enfermedad psiquiátrica grave.

Se incluyeron personas provenientes de diversas regiones de Colombia, teniendo en cuenta la multiculturalidad de este país (Rojas \& Castillo, 2005). Quedó integrada por 463 personas mayores de 60 años, de los cuales 200 (que equivalen al $43.1 \%$ de la muestra) viven en la región Andina (Santander y Norte de Santander), 180 (38.7\%) viven la Costa Caribe de Colombia (96 en la ciudad de Santa Marta y sus alrededores $=20.7 \%$ y 84 de Montería $=18.1 \%)$. También se evaluaron a 84 participantes $(18.1 \%)$ de la región de Antioquía y Medellín.

Instrumentos: El Cuestionario de Apoyo Social MOS es un cuestionario autoadministrado de 20 ítems. El primero indaga sobre el tamaño de la red de apoyo disponible y el resto se estructura en 4 subescalas que exploran 5 dimensiones funcionales del apoyo social: apoyo emocional/informacional (ítems 3, 4, 8, 9, 13, 16, 17 y 19), apoyo instrumental (ítems 2, 5, 12 y 15), interacción social positiva (ítems 7, 11, 14 y 18) y apoyo afectivo (ítems 6,10 y 20). Mediante una escala de 5 puntos, se pregunta con qué frecuencia está disponible para el entrevistado cada tipo de apoyo, con las siguientes opciones de respuesta: nunca, pocas veces, algunas veces, la mayoría de las veces, siempre.

La información sociodemográfica se obtuvo de una entrevista estructurada, diseñada especialmente para ese fin.

Procedimiento: Los cuestionarios fueron aplicados por estudiantes de Psicología de último semestre, previamente entrenados, quienes visitaron a los ancianos en sus hogares. La estrategia para reclutar a los participantes fue la de "bola de nieve" en la que una persona refiere a otra de sus mismas características. Se obtuvo el consentimiento informado de los participantes. Los datos pertenecen al estudio multicéntrico: "Factores psicosociales en el dolor osteomuscular crónico en adulto mayor. Un estudio transcultural", que fue aprobado por el Comité de Ética de las instituciones involucradas en el estudio. Para la presente investigación, sólo se tomaron en cuenta los datos de Colombia.

Análisis estadísticos: Para los análisis estadísticos se utilizaron los programas SPSS 25 y AMOS 25, con licencia de uso de la Universidad Cooperativa de Colombia.

Se investigaron las características sociodemográficas de la muestra (género, edad, convivencia con una pareja, vinculación laboral, nivel de estudios y región de procedencia) mediante el estudio de las distribuciones porcentuales y de frecuencia, y se obtuvo la estadística descriptiva de la variable continua (edad).

Se realizó la inspección de la medias y los coeficientes de asimetría de los ítems, así como de la magnitud de las correlaciones inter-ítem (mediante el coeficiente de correlación de Pearson) para decidir cuál era la matriz de asociación más adecuada para análisis factorial. Este análisis condujo a que se escogiera el método de Mínimos Cuadrados no Ponderados (ULS) que permite obtener estimadores que no responden a la hipótesis de normalidad de la distribución (González y Martín, 2012). Aunque se plantea que en general es un método de estimación poco utilizado, algunos autores como Valdivieso (2013) lo han utilizado con datos que no cumplen 
los requisitos exigidos para otros métodos. A esta estrategia de análisis se le señala problemas de precisión y estabilidad, por lo que se recomienda que se use sólo si las muestras son superiores a 200 (Lloret-Segura, Ferreres-Traver, Hernández-Baeza \& Tomás-Marco, 2014).

Según Ferrando y Lorenzo-Seva (2014), cuando se utiliza el ULS se debe evaluar el ajuste mediante indicadores que no dependan directamente de chi-cuadrado, ya que los indicadores de bondad de ajuste serán incorrectos y estarán generalmente inflados. Por esta razón, como índices para evaluar la bondad de ajuste se tuvieron en cuenta los que ofrece el programa AMOS para el método ULS, que son:

- Índice comparativo de ajuste (NFI): Mide la reducción proporcional en función de ajuste cuando se pasa del modelo nulo al modelo propuesto (Bentler \& Bonett, 1980). El rango de variación de este índice está entre 0 y 1 , se recomiendan valores superiores a 90 .

- GFI: es un estadístico de prueba sugerido por Jöreskog y Sörbom (1986), que señala la variabilidad explicada por el modelo, sus valores oscilan entre 0 (pobre ajuste) y 1 (perfecto ajuste). Valores superiores a .90 , indican un buen ajuste.

- AGFI: según Jöreskog y Sörbom (1986), ajusta el índice GFI por los grados de libertad del modelo propuesto y del modelo nulo. En la experiencia práctica, valores superiores a .90 son indicativos de un buen ajuste del modelo a los datos.

- Índice basado en residuo (root mean square residual- RMR): este índice se obtiene a través de la raíz cuadrada de los residuos al cuadrado, entre las matrices observadas y estimadas. Se utiliza normalmente con matrices de correlaciones ya que éstas no dependen de la unidad de medida. Al no tener en cuenta los grados de libertad, no se ha fijado un umbral para su interpretación Cuanto más pequeño sea el RMR, mejor. Una RMR de cero indica un ajuste perfecto. Se pueden considerar como aceptables, valores comprendidos entre .05 y .08 (Morata-Ramírez, Holgado-Tello, Barbero-García \& Méndez, 2015).

Además se calculó el alfa de Cronbach y se analizó el índice de homogeneidad corregido; y cómo cambia la fiabilidad de la escala y sub- escalas si se elimina cada ítem.

Por último, para identificar relaciones significativas atribuibles a las variables sociodemográficas investigadas (género y convivencia con una pareja) se compararon las puntuaciones obtenidas por los participantes en la escala total y en las subescalas mediante técnicas de análisis no paramétrico, más apropiadas para este tipo de datos que no cumplen requisitos de normalidad (Prueba U de Mann Withney).

Se consideró un nivel de significación inferior a .05

\section{Resultados}

\section{Caracterización de la muestra:}

La muestra del presente estudio estuvo conformada por 306 mujeres (66,1\%) y 157 hombres (33.9\%). La media en edad fue de 72.38 (mínimo $=60$, máximo $=98$, desviación típica $=8.18) ; 46.9 \%$ de los participantes se encontraban en el rango de edad de 60-69 años, 30.50\% se encontraban entre $71-80$ años; $15.33 \%$ entre $81-89$ años y $3.02 \%$ tenían entre 90 y más años de edad.

Del total de participantes, no tenían pareja el $57.5 \%$.

El $15.9 \%$ de los participantes (74 personas) dijeron que tenían un vínculo laboral, de los cuales reportaron que se trataba de un empleo estable 37 participantes ( $8 \%$ de la muestra total) y el resto dijo que trabajaban de manera esporádica o parcial.

114 personas $(24.7 \%)$ se encontraban jubilados o pensionados, $99(21.3 \%)$ se dedicaban a las tareas del hogar, $19(4.1 \%)$ a labores de beneficencia, $54(11.6 \%)$ no contestaron y el resto $(22.3 \%)$ estaba desvinculado del trabajo.

$60.3 \%$ participantes tenían educación básica; $23.3 \%$ educación media; $6.9 \%$ no tenían estudios y $9.3 \%$ participantes estudiaron educación superior.

Se encontraron diferencias significativas entre las regiones con respecto a la distribución de casos de acuerdo al sexo (Chi-cuadrado=11.87, $3 \mathrm{gl}$ ), pues en la muestra de Medellín se distribuyeron de manera equitativa ( $50 \%$ de hombres y $50 \%$ de mujeres), mientras que en las otras regiones predominaron las féminas $(69.8 \%$ en Magdalena, $70.2 \%$ en Montería y $69.3 \%$ en Santander). 
No se encontraron otras diferencias significativas entre las regiones con respecto a las otras variables sociodemográficas anteriormente descritas.

\section{Análisis de los items}

La Tabla 1 muestra la estadística descriptiva de los ítems. Como se observa, los 19 ítems de apoyo funcional tuvieron una desviación típica superior a 1 y los valores promedios estaban alrededor del punto medio de la escala. Nótese que la moda coincide con las puntuaciones máximas posibles para muchos ítems, y que la asimetría y curtosis son negativas.

Tabla 1: Estadísticos Descriptivos

\begin{tabular}{|c|c|c|c|c|c|c|c|c|c|c|c|c|c|c|c|c|c|c|c|c|}
\hline & & 2 & 3 & 4 & 5 & 6 & 7 & 8 & 9 & 10 & 11 & 12 & 13 & 14 & 15 & 16 & 17 & 18 & 19 & 20 \\
\hline & & $\mathrm{AI}$ & $\mathrm{AE}$ & $\mathrm{AE}$ & AI & AA & ISP & $\mathrm{AE}$ & $\mathrm{AE}$ & $\mathrm{AA}$ & ISP & $\mathrm{AI}$ & $\mathrm{AE}$ & ISP & $\mathrm{AI}$ & $\mathrm{AE}$ & $\mathrm{AE}$ & ISP & $\mathrm{AE}$ & $\mathrm{AA}$ \\
\hline \multirow[b]{2}{*}{$\mathrm{F}$} & & 4 & 456 & 456 & 456 & 4 & 4 & 4 & 456 & 456 & 456 & 456 & 456 & 456 & 456 & 556 & 56 & 56 & 456 & 456 \\
\hline & & 8 & 8 & 8 & 8 & 8 & 8 & 8 & 8 & 8 & 8 & 8 & 8 & 8 & 8 & 0 & 8 & 0 & 8 & 8 \\
\hline Media & 9 & 3.70 & 3.95 & 3.76 & 4.05 & 4.12 & 3.98 & 3.83 & 3.86 & 3.93 & 3.78 & 4.04 & 3.71 & 3.79 & 3.93 & 3.65 & 3.68 & 3.79 & 3.71 & 3.95 \\
\hline Mediana & 500 & 4.00 & 4.00 & 4.00 & 5.00 & 5.00 & 4.00 & 4.00 & 4.00 & 4.00 & 4.00 & 5.00 & 4.00 & 4.00 & 4.00 & 4.00 & 4.00 & 4.00 & 4.00 & 5.00 \\
\hline Moda & da & 5.00 & 5.00 & 5.00 & 5.00 & 5.00 & 5.00 & 5.00 & 5.00 & 5.00 & 5.00 & 5.00 & 5.00 & 5.00 & 5.00 & 5.00 & 5.00 & 5.00 & 5.00 & 5.00 \\
\hline Desv. típ. & 14.98 & 1.37 & 1.16 & 1.22 & 1.23 & 1.13 & 1.16 & 1.17 & 1.20 & 1.28 & 1.28 & 1.25 & 1.24 & 1.20 & 1.28 & 1.32 & 1.31 & 1.23 & 1.25 & 1.35 \\
\hline Asimetría & 6.6 & -.59 & -.75 & -.57 & -1.11 & -1.05 & -.88 & -.62 & -.68 & -.90 & -.7 & -1.06 & -.52 & -.5 & -.9 & -.50 & -.5 & -.62 & -.50 & -.95 \\
\hline Mínimo & .0 & 1.00 & 1.00 & 1.00 & 1.00 & 1.00 & 1.00 & 1.00 & 1.00 & 1.00 & 1.00 & 1.00 & 1.00 & 1.00 & 1.00 & 1.00 & 1.00 & 1.00 & 1.00 & 1.00 \\
\hline Máximo & 00.00 & 5.00 & 5.00 & 5.00 & 5.00 & 5.00 & 5.00 & 5.00 & 5.00 & 5.00 & 5.00 & 5.00 & 5.00 & 5.00 & 5.00 & 5.00 & 5.00 & 5.00 & 5.00 & 5.00 \\
\hline
\end{tabular}

a. Existen varias modas. Se mostrará el menor de los valores.

AE: Apoyo Emocional; AI: Apoyo Instrumental; AA: Apoyo Afectivo; ISP: Interacción Social Positiva. Las correlaciones entre los ítems estuvieron entre .45 a .74 , con más del $60 \%$ por encima de .50 .

El alfa de Cronbach para la escala total fue $=.96$ y también resultó alto para cada una de las subescalas (Escala Apoyo Emocional / Informacional= .94.; Escala Apoyo Instrumental= .86.; Escala Apoyo Afectivo=.89.; Escala de Interacción Social Positiva $=.90$.) y las correlaciones ítem-escala (Tabla 2) e ítems sub-escalas (Tabla 3) fueron superiores a .60 
Marta Martín-Carbonell, Ara Cerquera-Córdoba, Martha Fernández-Daza, Juan D. Higuita,

Gonzalo Galván Patrignani, Manuel Guerrero Martel y Antonio Riquelme Marín

Tabla 2: Correlaciones Ítem-Subescalas

\begin{tabular}{|c|c|c|c|c|c|c|}
\hline Subescalas & Ítems & $\begin{array}{l}\text { Media si se } \\
\text { elimina el } \\
\text { elemento }\end{array}$ & $\begin{array}{l}\text { Varianza si } \\
\text { se elimina el } \\
\text { elemento }\end{array}$ & $\begin{array}{l}\text { Correlación } \\
\text { elemento-total } \\
\text { corregida }\end{array}$ & $\begin{array}{l}\text { Correlación } \\
\text { múltiple al } \\
\text { cuadrado }\end{array}$ & $\begin{array}{l}\text { Alfa de Cronbach } \\
\text { si se elimina el } \\
\text { elemento }\end{array}$ \\
\hline \multirow{8}{*}{$\begin{array}{l}\text { Apoyo Emocional } \\
\text { Informacional }\end{array}$} & 3 & 26.24 & 57.71 & .74 & .59 & .94 \\
\hline & 4 & 26.43 & 57.01 & .74 & .60 & .94 \\
\hline & 8 & 26.35 & 56.98 & .78 & .63 & .94 \\
\hline & 9 & 26.33 & 55.83 & .82 & .69 & .94 \\
\hline & 13 & 26.48 & 54.85 & .85 & .75 & .93 \\
\hline & 16 & 26.54 & 54.09 & .84 & .73 & .93 \\
\hline & 17 & 26.51 & 53.77 & .86 & .79 & .93 \\
\hline & 19 & 26.48 & 55.23 & .82 & .69 & .94 \\
\hline \multirow{4}{*}{$\begin{array}{l}\text { Apoyo } \\
\text { Instrumental }\end{array}$} & 2 & 12.03 & 11.03 & 63 & .41 & .85 \\
\hline & 5 & 11.68 & 11,41 & .69 & .48 & .82 \\
\hline & 12 & 11.69 & 11.00 & .74 & .61 & .80 \\
\hline & 15 & 11.81 & 10.71 & .75 & .62 & .80 \\
\hline \multirow{3}{*}{$\begin{array}{l}\text { Apoyo } \\
\text { Afectivo }\end{array}$} & 6 & 7.89 & 6.06 & .80 & .65 & .85 \\
\hline & 10 & 8.07 & 5.38 & .81 & .67 & .83 \\
\hline & 20 & 8.05 & 5.21 & .78 & .61 & .87 \\
\hline \multirow{4}{*}{$\begin{array}{l}\text { Interacción Social } \\
\text { Positiva }\end{array}$} & 11 & 11.57 & 10.29 & .79 & .64 & .86 \\
\hline & 7 & 11.37 & 11.05 & .78 & .62 & .87 \\
\hline & 14 & 11.56 & 10.83 & .78 & .61 & .87 \\
\hline & 18 & 11.56 & 10.80 & .76 & .58 & .88 \\
\hline
\end{tabular}


Tabla 3: Correlaciones item- Escala Total

\begin{tabular}{llllll}
\hline Escala Total & $\begin{array}{l}\text { Media si se elimina } \\
\text { el elemento }\end{array}$ & $\begin{array}{l}\text { Varianza si } \\
\text { se elimina el } \\
\text { elemento }\end{array}$ & $\begin{array}{l}\text { Correlación ele- } \\
\text { mento-total cor- } \\
\text { regida }\end{array}$ & $\begin{array}{l}\text { Correlación múlti- } \\
\text { ple al cuadrado }\end{array}$ & $\begin{array}{l}\text { Alfa de Cronbach } \\
\text { si se elimina el } \\
\text { elemento }\end{array}$ \\
\hline 2AI & 69.61 & 326.10 & .63 & .47 & .96 \\
3AE & 69.36 & 325.31 & .77 & .67 & .96 \\
4AE & 69.55 & 326.06 & .72 & .62 & .96 \\
5AI & 69.26 & 327.87 & .66 & .59 & .96 \\
6AA & 69.19 & 324.86 & .80 & .77 & .96 \\
7ISP & 69.32 & 323.96 & .81 & .73 & .96 \\
8AE & 69.47 & 324.47 & .79 & .70 & .96 \\
9AE & 69.44 & 322.94 & .80 & .71 & .96 \\
10AA & 69.38 & 320.93 & .80 & .74 & .96 \\
11 ISP & 69.53 & 320.34 & .81 & .72 & .96 \\
12AI & 69.26 & 326.00 & .70 & .66 & .96 \\
13AE & 69.60 & 321.63 & .80 & .79 & .96 \\
14ISP & 69.52 & 322.60 & .81 & .73 & .96 \\
15AI & 69.38 & 323.86 & .73 & .67 & .96 \\
16AE & 69.66 & 318.80 & .82 & .76 & .96 \\
17AE & 69.63 & 319.29 & .81 & .80 & .96 \\
18ISP & 69.52 & 323.51 & .77 & .68 & .96 \\
19AE & 69.60 & 320.51 & .82 & .77 & .96 \\
\hline
\end{tabular}

Nota: AE: Apoyo Emocional; AI: Apoyo Instrumental; AA: Apoyo Afectivo; ISP: Interacción Social Positiva.

\section{Estudio de la estructura factorial:}

Se evaluaron 3 modelos: un modelo de un factor (dada la alta consistencia interna hallada en el análisis de fiabilidad de la escala total); y los modelos de 3 y 4 factores correlacionados teniendo en cuenta los estudios de validación del MOS en población colombiana. Los resultados se sintetizan en la Tabla 4. Aunque en general, todos los modelos estudiados ofrecen buenos índices de ajuste, el modelo de 4 factores correlacionados fue el que mejores índices obtuvo.

Tabla 4: Resumen del AFC (Método de Mínimos Cuadrados no Ponderados - ULS)

\begin{tabular}{lcccc}
\hline \multicolumn{1}{c}{ Modelo } & NFI & AGFI & RMR & NFI \\
\hline Un solo factor & .992 & .991 & .86 & .991 \\
$\begin{array}{l}4 \text { Factores } \\
\text { correlacionados }\end{array}$ & .997 & .996 & .56 & .996 \\
$\begin{array}{l}\text { 3 Factores } \\
\text { correlacionados }\end{array}$ & .996 & .995 & .62 & .995 \\
\hline
\end{tabular}

La figura 1 ilustra el modelo de 4 factores relacionados, obsérvese que los factores están integrados por los mismos ítems que propusieron Sherbourne y Stewart (1991) en el estudio original. 


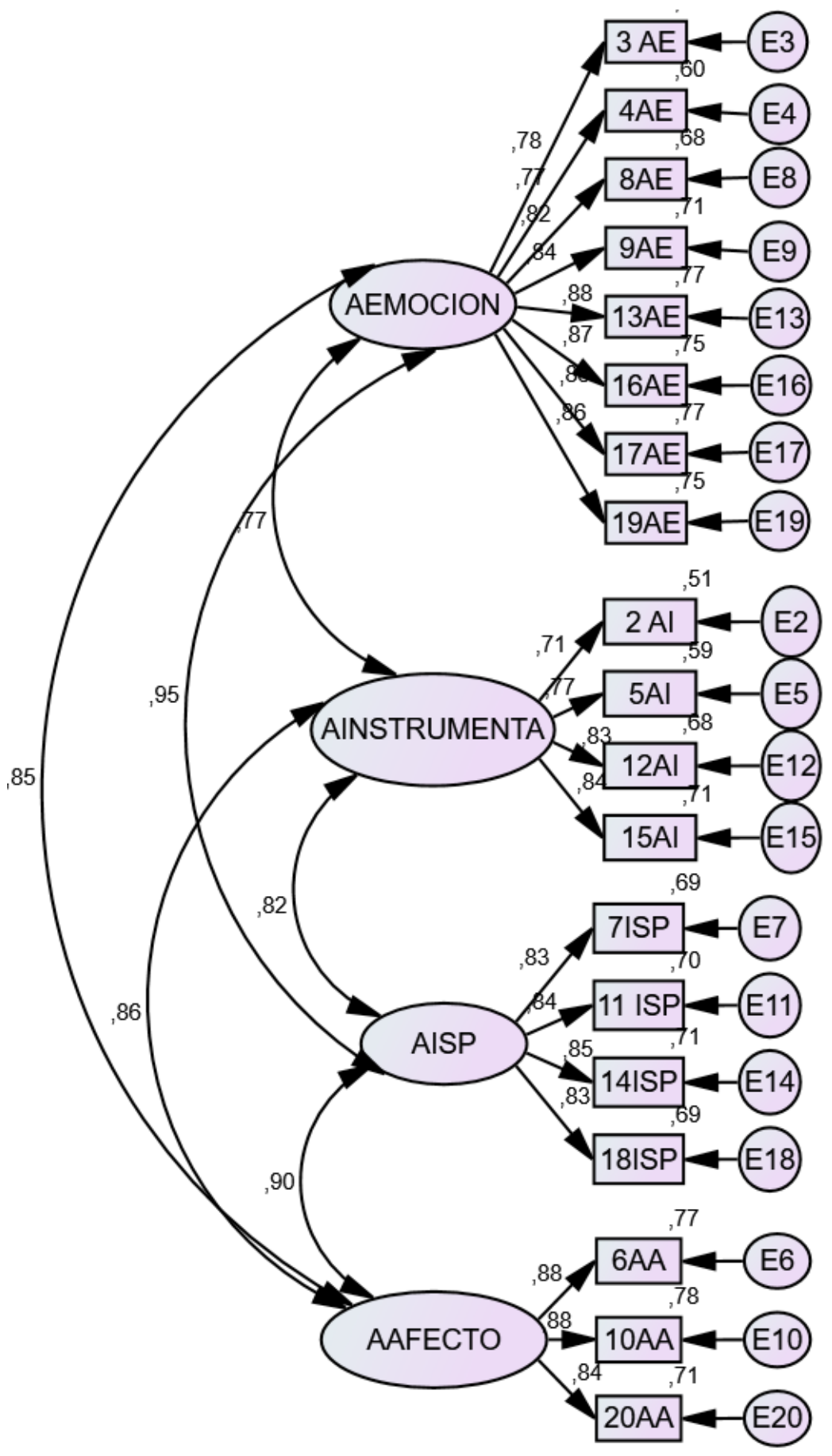

Figura 1: Modelo de 4 factores correlacionados

Dimensiones del apoyo y variables sociodemográficas:

La tabla 5 muestra los resultados de los estadígrafos que valoran la significación de las diferencias en las puntuaciones en las diferentes sub-escalas del MOS entre personas de diferente género y convivencia con una pareja. 
Con respecto al género, se detectaron diferencias significativas para el apoyo global y para todas las dimensiones del apoyo, menos para la instrumental, siendo en todos los casos las mujeres quienes reciben más apoyo.

Las personas que disponían de relación de pareja también obtuvieron más apoyo instrumental, más interacción social positiva, más apoyo afectivo y más apoyo global, pero no se detectaron diferencias significativas para la subescala de apoyo emocional/informacional.

Tabla 5: Diferencias por sexo y pareja.

\begin{tabular}{lllllll}
\hline & & AE & AI & ISP & AA & Total \\
\hline Diferencias por Sexo. & U de Mann-Whitney & 19966.50 & 22059.50 & 19536.00 & 19167.00 & 19836.50 \\
& W de Wilcoxon & 31747.50 & $33994, .50$ & 31471.00 & 31102.00 & 31617.50 \\
& Z & -2.38 & -1.02 & -2.93 & -3.29 & -2.47 \\
& Sig. asintót. (bilateral) & .017 & .304 & .003 & .001 & .013 \\
Diferencias relaciona- & U de Mann-Whitney & 22384.50 & 22008.00 & 21987.50 & 20244.50 & 21381.50 \\
das con tener pareja & W de Wilcoxon & 56575.50 & 56724.00 & 56703.50 & 54960.50 & 55572.50 \\
& Z & -2.04 & -2.56 & -2.56 & -3.92 & -2.76 \\
& Sig. asintót. (bilateral) & .041 & .010 & .010 & .000 & .006 \\
\hline
\end{tabular}

\section{Discusión}

La razón principal del presente estudio fue examinar la estructura del Cuestionario de Apoyo Social MOS (Sherbourne y Stewart, 1991) para la evaluación de ancianos colombianos con dolor osteomuscular crónico.

Diversos autores plantean que elegir el método adecuado en la fase del AFC de estimación de parámetros influye en la fase posterior de evaluación del ajuste del modelo teórico especificado (Flores y Medrano, 2016; Lloret et al. 2014), ya que el AFC exige el cumplimiento de ciertos supuestos, siendo la asimetría en la distribución de las variables observadas uno de los principales aspectos que distorsionan estos supuestos. A pesar de ello, lo habitual ha sido tratar las variables observadas como si fueran continuas, por lo que el método de estimación de Máxima Verosimilitud (ML) ha tenido un uso muy extendido (Morata-Ramírez, Holgado-Tello, BarberoGarcía \& Méndez, 2015). Para nuestro estudio elegimos el método ULS ya que se encontró que los datos no cumplían los supuestos que se exige para el método de máxima verosimilitud, y se corroboró que la estructura de 4 factores correlacionados obtiene los mejores índices de ajuste, coincidiendo con los resultados de Londoño et al. (2012) en su estudio previo con población general colombiana; y también con los hallazgos de las autoras de la versión original. Esta estructura factorial ha sido corroborada en poblaciones muy diversas como ancianos chinos (Yu, Lee \& Woo, 2004), enfermos crónicos italianos (Giangrasso \& Casale, 2014), mujeres víctimas de delitos (Higgins, Marcum, Golder, Hall \& Logan, 2015), o pacientes malayos con VIH (Saddki et al., 2017).

Sin embargo, también se obtuvo índices aceptables para los otros modelos porque aunque existe suficiente evidencia empírica de la multidimensionalidad del apoyo social, algunos autores consideran que los componentes de apoyo social están demasiado correlacionados para ser distinguidos empíricamente (Sherbourne \& Stewart, 1991); y de hecho, en nuestro estudio, se encontraron altas correlaciones entre factores . Esto se puede explicar también porque con frecuencia, una misma persona proporciona diversos tipos de apoyo.

Otra evidencia de las buenas propiedades psicométricas del MOS se obtuvo del análisis de los valores de media y desviación típica de los ítems, que sugiere que tienen un adecuado poder de discriminación (CarreteroDios \& Pérez, 2005).

También podría interpretarse favorablemente en términos de validez de la estructura, los altos índices de Cronbach de la escala total y subescalas, así como altas correlaciones ítems-escalas. Estos resultados son similares a los descritos en otros estudios realizados en Latinoamérica (Baca, 2016).

A favor de que las dimensiones reflejan como las personas vivencian el apoyo en la realidad, están las diferencias encontradas con respecto a su asociación con el género. La literatura es consistente acerca de que en la cultura occidental las mujeres tienen redes sociales mayores y más complejas, con mayor nivel de comunicación 
y multiplicidad, involucrando relaciones con familias, amigos y vecinos. Los hombres se afectan más por la pérdida de contactos sociales asociada a la jubilación. Así mismo, las mujeres son más dadas a recibir y proveer apoyo emocional, informacional y financiero, incluyendo el de la familia y la pareja y también se benefician más del apoyo (Harling, Morris, Manderson, Perkins \& Berkman, 2017).

Igualmente, existe consenso acerca de que las personas con pareja perciben y reportan más apoyo, especialmente en la vejez (Riquelme et al. 2016), lo que coincide con los resultados de esta investigación.

Una limitación de este estudio es la estrategia utilizada para seleccionar los casos, lo que se debió a que no se disponía de los recursos necesarios. A pesar de ello, resulta interesante que desde el punto de vista sociodemográfico, la muestra no difiere de lo que se considera que es el patrón para Colombia (Flores, Villar, Puerta \& Berrocal, 2015; Méndez, 2016; Ministerio de Salud y Protección Social, 2013).

Otra limitación es que no se evaluaron otras medidas del apoyo social para examinar la validez concurrente del MOS y/o servir como "regla de oro" para determinar puntos de corte que faciliten su interpretación de cara a las intervenciones clínicas y psicosociales, lo que deberá ser objeto de próximas investigaciones.

Mientras tanto, esta investigación puede ser un referente para sustentar la utilidad y validez del cuestionario MOS para la evaluación de la funcionalidad del apoyo social percibido por ancianos colombianos con dolor crónico.

\section{Referencias}

Acitelli, L. K., \& Antonucci, T. C. (1994). Gender differences in the link between marital support and satisfaction in older couples. Journal of personality and social psychology, 67(4), 688.

Akar, A. (2013). Diseño y evaluación de un modelo de intervención en salud mental temprana para el fortalecimiento del vínculo madre-bebe en una policlínica de primer nivel de atención en Montevideo. En V Congreso Internacional de Investigación y Práctica Profesional en Psicología. XX Jornadas de Investigación Noveno Encuentro de Investigadores en Psicología del MERCOSUR. Facultad de Psicología-Universidad de Buenos Aires.

Alaloul, F., \& Hall, L. (2016, March). Examination Of The Validity Of The Arabic Version Of The Medical Outcome Study (MOS) Social Support Survey Using Confirmatory Factor Analysis. In Oncology Nursing Forum, 43 (2), 128.

Alonso, F. A., Montes, M. A., Menéndez, V.C., \& Pereira, M.G. (2006). Cultural adaptation and validation of the medical outcomes study social support survey questionnaire (MOS-SSS). Acta médica portuguesa, 20(6), 525-534.

Alva, A. (2016). Nivel de apoyo social percibido en la familia por la adolescente embarazada usuaria de un Hospital General de Lima Metropolitana 2015 (tesis de pregrado). Universidad Nacional Mayor de San Marcos, Lima, Perú.

Anderson, D., Bilodeau, B., Deshaies, G., Gilbert, M., \& Jobin, J. (2005). French-Canadian validation of the MOS social support survey. The Canadian journal of cardiology, 21(10), 867-873.

Antonucci, T.C., \& Akiyama, H. (1987). An examination of sex differences in social support among older men and women. Sex roles, 17(11-12), 737-749.

Baca, D. (2016). Confiabilidad y validez del cuestionario de apoyo social en pacientes con cáncer de Trujillo. Revista de Investigación en Psicología, 19(1), 177-190.

Bengtson, V. (2018). Global aging and challenges to families. New York, E.E.U.U.: Routledge.

Bentler, P. M. \& Bonett, D. G. (1980). Significance tests and goodness of fit in the analysis of covariance structures. Psychological Bulletin, 88, 588-606.

Carretero-Dios, H., \& Pérez, C. (2005). Normas para el desarrollo y revisión de estudios instrumentales. International Journal of clinical and health psychology, 5(3), 521-551.

Castillo, L. (2016). Apoyo social y su relación con las conductas sexuales de riesgo de adolescentes, Institución educativa Víctor Raúl. Victor Larco, 2016 (tesis de pregrado). Universidad Privada Antenor Orrego, Trujillo, Perú.

Costa Requena, G., Salamero, M., Gil, F. (2007).Validación del cuestionario MOS-SSS de apoyo social en pacientes con cáncer. Medicina Clínica, 128 (687), 91.41.

Cross-Denny, B., \& Robinson, M. A. (2017). Using the Social Determinants of Health as a Framework to Examine and Address Predictors of Depression in Later Life. Ageing International, 42(4), 393-412. DOI: 10.1007/s12126-017-9278-6

De la Revilla Ahumada, L., Luna del Castillo, J., Bailón Muñoz, E., y Medina Moruno, I. (2005). Validación del cuestionario MOS de apoyo social en atención primaria. Medicina de Familia (And), 6 (1), 10-18. 
Dumitrache, C.G., Rubio, L., \& Rubio-Herrera, R. (2017). Perceived health status and life satisfaction in old age, and the moderating role of social support. Aging \& mental health, 21(7), 751-757.

Ferrando, P \& Lorenzo-Seva, U. (2014). El análisis factorial exploratorio de los ítems: algunas consideraciones adicionales. Anales de Psicología, 2014, 30(3), 170-1175. http://dx.doi.org/10.6018/analesps.30.3.199991

Flores, P.E., \& Medrano, L.A. (2016). El afecto y sus dimensiones: Modelos contrastados mediante análisis factorial confirmatorio de la Escala PANAS. Liberabit, 22(2), 173-184.

Flórez, C., Villar, L., Puerta, N y Berrocal, L. (2015). El proceso de envejecimiento de la población en Colombia: 1985-2050. Bogotá, Colombia: Editorial Fundación Saldarriaga Concha.

Galindo, B. V. (2011). Satisfacción y apoyo social familiar en una muestra de personas con Trastorno Mental Grave (trabajo de pregrado). Universidad Castilla La Mancha, Cuenca, España.

Giangrasso, B., \& Casale, S. (2014). Psychometric Properties of the Medical Outcome Study Social Support Survey with a General Population Sample of Undergraduate Students. Social Indicators Research, 116(1), 185-197. http://doi.org/10.1007/s11205-013-0277-z

Gonzales, H. (2011). Apoyo social y calidad de vida en adultos mayores institucionalizados en el hogar Santa Teresa se Jornet (tesis de pregrado). Universidad Autónoma Juan Misael Saracho, Tarija, Bolivia

González, N. y Martín, R. (2012). Introducción a la modelización con estructuras de covarianzas en ciencias sociales: uso del programa AMOS. Recuperado de https://documentslide.org/the-philosophy-of-money.html?utm_source=introduccion-a-la-modelizacion-con-estructuras-de-covarianzas-en-ciencias-sociales-uso-del-programa-amos.

González, N. \& Estévez, A (2017). El apoyo social percibido moderador entre el uso problemático de Internet y la sintomatología depresiva en jóvenes adultos. Health and Addictions/Salud y Drogas, 17(1), 53-62.

Griep, R.H., Chor, D., Faerstein, E., Werneck, G. L., \& Lopes, C. S. (2005). Validade de constructo de escala de Apoio Social do Medical Outcomes Study adaptada para o português no estudo Pró-Saúde (Construct validity scale of Social Support from the Medical Outcomes Study adapted to Portuguese in Pro study-Health). Cadernos de Saúde Pública, 21(3), 703-714.

Harling, G., Morris, K. A., Manderson, L., Perkins, J.M., \& Berkman, L. (2017). Social Networks and Social Support among Older Adults in Rural South Africa: Findings from the Health and Aging in Africa: A Longitudinal Study of an INDEPTH Community in South Africa. Recuperado de https://papers.ssrn.com/sol3/papers.cfm?abstract id=2977867

Hernández, R.L., \& Ramírez, M. T. (2014). Apoyo social en mujeres de familias monoparentales y biparentales. Psicología y salud, 16(2), 149-157.

Herrero, J. (2010). El Análisis Factorial Confirmatorio en el estudio de la Estructura y Estabilidad de los Instrumentos de Evaluación: Un ejemplo con el Cuestionario de Autoestima CA-14. Intervención Psicosocial. 19(3), 289-300.

Higgins, G., Marcum, C.D., Golder, S., Hall, M. T., \& Logan, T.K. (2015). Confirmatory Factor Analysis of the Medical Outcomes Study-Social Support Survey: Examining the Factor Structure Among Victimized Women on Probation and Parole. American Journal of Criminal Justice, 40(4), 811-822. http://doi.org/10.1007/s12103-015-9290-x

Holden, L., Lee, C., Hockey, R., Ware, R. S., \& Dobson, A. J. (2014). Validation of the MOS Social Support Survey 6-item (MOS-SSS-6) measure with two large population-based samples of Australian women. Quality of Life Research, 23(10), 2849-2853.

House, J. S. \& Kahn, R. L. (1985). Measures and concepts of social support. En S. Cohen \& L. S. Syme. Social Support and Health (pp. 83-108). London: Academic Press.

Joreskog, K. G., \& Sorbom, D. (1986). LISREL VI: Analysis of linear structural relationships by maximum likelihood, instrumental variables, and least squares methods. Mooresville, IN: Scientific Software, Inc.

Lee, J.E., Kahana, B., \& Kahana, E. (2016). Social support and cognitive functioning as resources for elderly persons with chronic arthritis pain. Aging \& mental health, 20(4), 370-379.

Lloret-Segura, S., Ferreres-Traver, A., Hernández-Baeza, A., \& Tomás-Marco, I. (2014). El análisis factorial exploratorio de los ítems: una guía práctica, revisada y actualizada. Anales de Psicología, 30(3), 1151-1169.

Londoño, N. E., Rogers, H., Castilla, J.F., Posada, S., Ochoa, N., Jaramillo, M. A., Oliveros, M., Palacio, J., Aguirre, D. (2012). Validación en Colombia del cuestionario MOS de apoyo social. International Journal of Psychological Research, 5(1), 142-150.

Lu, M.H., Wang, G.H., Lei, H., Shi, M.L., Zhu, R., \& Jiang, F. (2018). Social Support as Mediator and Moderator of the Relationship Between Parenting Stress and Life Satisfaction Among the Chinese Parents of Children with ASD. Journal of autism and developmental disorders, 48(4), 1181-1188.

Lyyra, T. M., \& Heikkinen, R. L. (2006). Perceived social support and mortality in older people. The Journals of Gerontology Series B: Psychological Sciences and Social Sciences, 61(3), S147-S152.

Martínez Basurto, A. E., Sánchez Román, S., Aguilar Villalobos, E. J., Rodríguez Pérez, V., \& Riveros Rosas, A. (2014). Adaptación y Validación del Cuestionario MOS de Apoyo Socialen pacientes mexicanos con VIH+. Revista Latinoamericana de Medicina Con- 
ductual/Latin American Journal of Behavioral Medicine, 4(2), 93-101.

Martínez, B. B., Buz, J., Navarro, A. B., \& López-Martín, L. (2017). Equidad en el apoyo social recíproco y satisfacción vital en personas mayores. Universitas Psychologica, 16(1), 146-158.

Matos, M., Bernardes, S. F., Goubert, L., \& Beyers, W. (2017). Buffer or amplifier? Longitudinal effects of social support for functional autonomy/dependence on older adults' chronic-pain experiences. Health Psychology, 36(12), 1195. DOI: 10.1037/hea0000512.

Méndez, F. (2016). Encuesta Nacional de salud, Bienestar y Envejecimiento 2015. Presentación de resultados.

Ministerio de Salud y Protección Social Colombia (2013). Envejecimiento demográfico. Colombia 1951-2020. Dinámica demográfica y estructuras poblacionales. Recuperado de: https://www.minsalud.gov.co/sites/rid/Lists/BibliotecaDigital/RIDE/DE/PS/

Morata-Ramírez, M. A., Holgado-Tello, F., Barbero-García, I \& Méndez, G. (2015). Análisis factorial confirmatorio: recomendaciones sobre mínimos cuadrados no ponderados en función del error Tipo I de Ji-Cuadrado y RMSEA. Acción Psicológica, 12(1), 79-90. https://dx.doi.org/doi.org/10.5944/ap.12.1.14362

Moreta Telenchana, A. (2016). Apoyo Social percibido y autoestima de los Adolescentes en acogimiento Institucional Emergente (tesis de pregrado). Universidad Técnica de Ambato, Ambato, Ecuador.

Muñoz Hernández, A. M. (2016). El apoyo social y su influencia en los síntomas de trastorno de estrés postraumático con depresión en víctimas de desastres naturales (tesis de pregrado). Universidad San Francisco de Quito, Quito, Ecuador.

Núñez, A.S (2015). Análisis del apoyo social percibido en pacientes renales del Instituto de Previsión Social. Revista ScientiAmericana, 2(2), 1-20.

Orcasita, L. T., Peralta, A., Valderrama, L., \& Uribe, A. F. (2010). Apoyo social y conductas de riesgo en adolescentes diagnosticados y no diagnosticados con VIH/SIDA en Cali-Colombia. Revista Virtual Universidad Católica del Norte, 31, 155-195.

Orcasita, L. T., Uribe, A. F., Castellanos, L.P., \& Gutiérrez, M. (2012). Apoyo social y conductas sexuales de riesgo en adolescentes del municipio de Lebrija-Santander. Revista de Psicología (PUCP), 30(2), 371-406.

Ordoñez Torres, D. L \& Tapia Garzón, M.E. (2016). Asociación entre el nivel de apoyo social y familiar con la adherencia terapéutica en pacientes diagnosticados de hipertensión arterial que acuden a la consulta externa del Centro de Salud Augusto Egas distrito 23 de Octubre a diciembre del 2016 (tesis de especialidad). Pontificia Universidad Católica de Ecuador, Santo Domingo, Ecuador.

Palmar, A. \& Pico, A. (2017) Exploración de la estructura del MOS en ancianos con dolor osteomuscular del Magdalena (tesis de grado) Universidad Cooperativa de Colombia, Santa Marta.

Poblete, F., Glasinovic, A., Sapag, J., Barticevic, N., Arenas, A., \& Padilla, O. (2015). Apoyo social y salud cardiovascular: adaptación de una escala de apoyo social en pacientes hipertensos y diabéticos en la atención primaria chilena. Atención Primaria, 47(8), 523-531

Priede, A., Andreu, Y., Martínez, P., Conchado, A., Ruiz-Torres, M., \& González-Blanch, C. (2018). The factor structure of the Medical Outcomes Study-Social Support Survey: A comparison of different models in a sample of recently diagnosed cancer patients. Journal Of Psychosomatic Research, 108, 32-38.

Riquelme-Marín, A., Martín-Carbonell M., Ortigosa-Quiles, J. M, Meda-Lara, R. M., Pérez-Díaz, R, \& López-Navas, A. I (2016). Implications of age and country of membership in the relationship between perceived emotional support and depression: A cross-cultural study | Implicaciones de la edad y el país de pertenencia en la relación entre apoyo emocional percibido y depresión: Un. Anales de Psicología, 32(2), 393-404. http://doi.org/10.6018/analesps.32.2.203611

Rodríguez Espínola, S. (2011). Adaptación a niños del cuestionario MOS de apoyo social percibido. Liberabit, 17(2), 117-128.

Rojas, A y Castillo, E. (2005). Educar a los otros. Estado, políticas educativas y diferencia cultural en Colombia. Popayán, Colombia: Editorial Universidad del Cauca.

Rosa-Rodríguez, Y., Negrón, C.N., Maldonado P.Y., Toledo O.N., \& Quiñones B. A. (2015). Dimensiones de bienestar psicológico y apoyo social percibido con relación al sexo y nivel de estudio en universitarios. Avances en Psicología Latinoamericana, 33(1), 31-43. doi: dx.doi.org/10.12804/ap133.01.2015.03

Saddki, N., Sulaiman, Z., Abdullah, S., Zakaria, N., Mohamad, N., Ab Razak, A., \& Zainan Abidin, Z. (2017). Psychometric properties of the Malay version of the Medical Outcomes Study Social Support Survey (MOS-SSS) in a sample of patients with HIV. Journal of HIV/AIDS \& Social Services, 16(1), 60-74. JOUR. http://doi.org/10.1080/15381501.2015.1107801

Schwartz, E., \& Litwin, H. (2018). Social network changes among older Europeans: the role of gender. European Journal of Ageing, 1-9. DOI https://doi.org/10.1007/s10433-017-0454-z

Sherbourne, C. y Stewart, A. (1991). The MOS Social Support Survey. Social Science \& Medicine, 32, 705-714.

Shyu, Y.I., Tang, W. R., Liang, J., Weng L. J. (2006). Psychometric testing of the social support survey on a Taiwanese sample. Nursing research, 55(6), 411-417.

Soares, A., Biasoli, I., Scheliga, A., Baptista, R., Brabo E., Morais,...Spector, N. (2012). Validation of the Brazilian Portuguese version of the medical outcomes study-social support survey in Hodg-kin's lymphoma survivors. Support Care Cancer, 20(8), $1895-1900$. 
Tajvar, M., Grundy, E., \& Fletcher, A. (2018). Social support and mental health status of older people: a population-based study in Iran-Tehran. Aging \& mental health, 22(3), 344-353.

Terol, M., López, S., Neipp, M., Rodríguez, J., Pastor, M. A., Martín-Aragon, M. (2004). Apoyo social e instrumentos de evaluación: revisión y clasificación. Anuario de psicología/The UB Journal of psychology, 35(1), 23-46.

Trejos, A. M., Mosquera, M., \& Tuesca, R. (2009). Niñez afectada con VIH/SIDA: Calidad de vida, funcionalidad familiar y apoyo social en cinco ciudades colombianas. Revista Salud Uninorte, 25(1), 17-32.

Uchino, B. N. (2009). Understanding the links between social support and physical health: A life-span perspective with emphasis on the separability of perceived and received support. Perspectives on Psychological Science, 4(3), 236-255.

Uribe, A.F. (2016). Salud sexual, apoyo social y funcionamiento familiar en universitarios. Revista Virtual Universidad Católica del Norte, 49, 206-229.

Uribe, A.F., Orcasita, L., \& Aguillón Gómez, E. (2012). Bullying, redes de apoyo social y funcionamiento familiar en adolescentes de una institución educativa de Santander, Colombia. Psychologia. Avances de la disciplina, 6(2), 83-99.

Valdivieso, C. (2013). Efecto de los métodos de estimación en las modelaciones de estructuras de covarianzas sobre un modelo estructural de evaluación del servicio de clases. Comunicaciones en Estadística, 6 (1), 21-44.

Wang, W., Zheng, X., He, H. G., \& Thompson, D. R. (2013). Psychometric testing of the Chinese Mandarin version of the Medical Outcomes Study Social Support Survey in patients with coronary heart disease in mainland China. Quality of Life Research, 22(8), $1965-1971$.

Yu, D. S., Lee, D. T., \& Woo, J. (2004). Psychometric testing of the Chinese version of the medical outcomes study social support survey (MOS-SSS-C). Research in nursing \& health, 27(2), 135-143. http://doi.org/10.1002/nur.20008 